\title{
Performance Evaluation and Fusion of Methods for Early Detection of Alzheimer Disease
}

\author{
Brahim Hamadicharef, Cuntai Guan \\ Institute for Infocomm Research $\left(\mathrm{I}^{2} \mathrm{R}\right)$ \\ 21 Heng Mui Keng Terrace, Singapore 119613 \\ \{bhamadi;ctguan\}@i2r.a-star.edu.sg \\ Nigel Hudson \\ Department of Neurophysiology \\ Derriford Hospital, Plymouth PL1, U.K. \\ Nigel.Hudson@phnt.swest.nhs.uk
}

\author{
Emmanuel C. Ifeachor \\ University of Plymouth \\ Drake Circus, Plymouth PL4 8AA, U.K. \\ eifeachor@plymouth.ac.uk \\ Sunil Wimalaratna \\ Department of Neurology \\ Radcliffe Infirmary, Oxford OX2 6HE, U.K. \\ wim@doctors.org.uk
}

\begin{abstract}
The number of people that develop Alzheimer's Disease $(A D)$ is rapidly rising, while the initial diagnosis and care of $A D$ patients typically falls on non-specialist and still taking up to 3-5 years before being referred to specialists. An urgent need thus exists to develop methods to extract accurate and robust biomarkers from low-cost and non intrusive modalities such as electroencephalograms (EEGs). Contributions of this paper are three-fold. First we review 8 promising methods for early diagnosis of $A D$ and undertake a performance evaluation using ROC analysis. We find that fractal dimension $(A U C=0.989)$, zero crossing interval $(A U C=0.980)$ and spectrum analysis of power alpha/theta ratio $\left(P w r_{\alpha, \theta}\right)(A U C=0.975)$ perform best. with all three having sensitivity and specificity higher than $94 \%$. We plot ROC curve with $95 \%$ confidence contours because of the small size of our data set (17 AD and 24 NOLD). Second, we investigate a fusion approach to combine these methods, using a logistic regression model, into one single more accurate biomarker $(A U C=1.0)$. Thirdly, to help support the distribution and use of these methods for early detection and care of $A D$, we developed them as web-services, integrated into online tools available from the BIOPATTERN project portal (www.biopattern.org).
\end{abstract}

\section{Introduction}

Worldwide, the number of people that develop Alzheimer's Disease (AD) is rapidly rising. In 2000 there were, in the US, 4.5 million persons with $\mathrm{AD}$ with this number likely to increase to 13.2 million by 2050 [17]. Figures for Europe are also alarming, the number of prevalent patients with dementia in 2000 was 7.1 million and likely to rise, based on the population projections of the United Nations, to about 16.2 million within the next 50 years [32]. This is creating considerable financial burden on the health and social services [26] and making the clinical and economic benefits of early diagnosis an important issue [25]. In the long term, the real cost [7] and economic impact [4][31] of such disease is rather difficult to assess.

The initial diagnosis and care of AD patients typically falls on non-specialist, e.g. General Practitioners (GPs) at a surgery, before they are referred to specialists (e.g. in geriatric neurology) for further tests, and could take up to 3-5 years [8][27].

An urgent need is therefore required to develop methods to extract accurate and robust biomarkers from, low-cost and non intrusive, electroencephalograms (EEGs). There is also a need develop computer tools that can, easily and accurately, help early diagnosis within an acceptable time frame.

Biomarkers are found useful in many aspects of AD research. First, they are used as index to distinguish Normal Old (NOLD) persons from patients with Mild Cognitive Impairment (MCI) or probable AD [5]. Second, they are used monitor the progression of the disease in serial EEG studies [30]. Finally, they are used to assess the effectiveness of drug treatments in clinical trials [6]. In our research we focus on EEG-based biomarkers but it is worth mentioning that recent research aim to exploit multiple modalities [14][9].

The aims of our research, presented in this paper, are threefold: 1) to review promising methods for early diagnosis of $\mathrm{AD}$ and undertake a performance evaluation using 
ROC analysis, 2) to investigate a fusion approach to combine these methods into one single biomarker, and 3) to help support the distribution and use of these methods with online tools developed as web-services for early detection and care of $\mathrm{AD}$ [15].

The remainder of the paper is organized as follows. In Section 2, we review the methods used in the evaluation study. In Section 3, we describe the EEG data set. Results are presented in Section 4. Finally, in Section 5, we conclude the paper.

\section{Methods}

In this paper, we restrict the evaluation to the following methods: spectrum analysis with power ratio $\left(P w r_{\alpha, \theta}\right)$, fractal dimension (FD), zero-crossing interval (ZCI), central tendency measure (CTM), HjorthIndex a measure combining activity, mobility and complexity, sample entropy [2], Kolmogorov complexity [24], Tsallis entropy (TsaEnt) [34].

Power spectrum analysis is the reference method used for research in AD [29][28][10]. We filter each EEG channel to extract frequency bands, typically delta $(\delta, 2-4 \mathrm{~Hz})$, theta $(\theta, 4-8 \mathrm{~Hz})$ and alpha $(\alpha, 8-13 \mathrm{~Hz})$, and beta $(\beta, 13-$ $30 \mathrm{~Hz}$ ). The power ratio, $P w r_{\alpha, \theta}$, is then calculated as:

$$
\text { Pwr }_{\alpha, \theta}=\frac{\text { Power }_{\alpha}}{\text { Power }_{\alpha}+\text { Power }_{\theta}}
$$

Sophisticated nonlinear signal processing techniques now exist that can be used to handle problems associated with, for example, non-linearity and non-stationarity [22]. A good review of method for $\mathrm{AD}$ can be found in [23]. We use FD and ZCI that were the basis of previous work [19][18].

CTM has also been used for early detection of AD [3]. The method allows to quantify the variability seen in the second order difference plot and is computed by selecting a circular region of radius $r$, around the origin, and then counting the number of points falling within the radius, over the total number of points. CTM is defined as:

$$
\begin{gathered}
C T M=\frac{\sum_{i=1}^{n-2} \delta\left(d_{i}\right)}{n-2} \\
\delta\left(d_{i}\right)= \begin{cases}1 & \text { if }\left(\left[x_{21}\right]^{2}+\left[x_{10}\right]^{2}\right)^{0.5}<r \\
0 & \text { otherwise }\end{cases}
\end{gathered}
$$

where $x_{21}=\left[x_{i+2}-x_{i+1}\right]$ and $x_{10}=\left[x_{i+1}-x_{i}\right]$. For sample entropy we use the same setting as in [2] with $\mathrm{m}=1$ and $r=0.25$ times the standard deviation of the EEG channel. Finally, we a Hjorth-based index [12] using parameters activity, mobility and complexity [20] defined as:

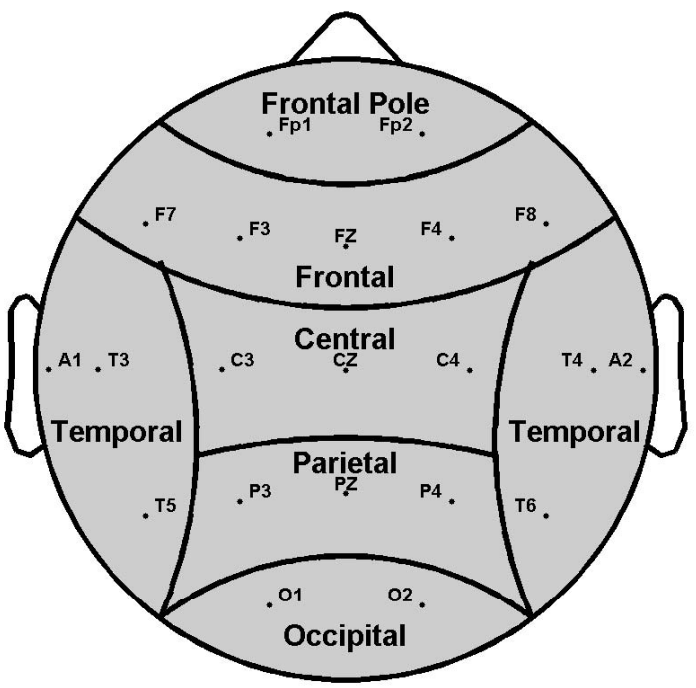

Figure 1. Electrodes and brain regions

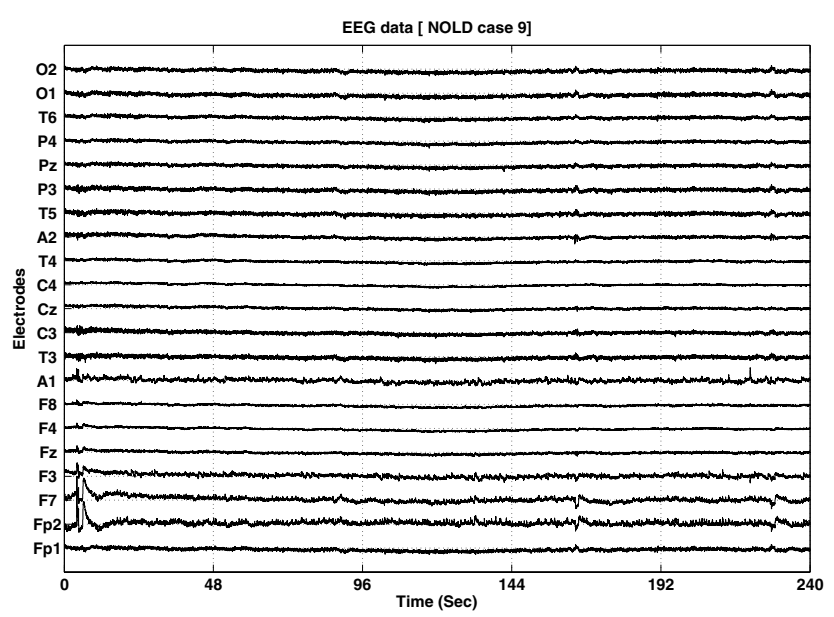

Figure 2. Example of EEG trace

$$
\begin{gathered}
\operatorname{Activity}(x(t))=\operatorname{VAR}(x(t)) \\
\operatorname{Mobility}(x(t))=\sqrt{\frac{\operatorname{Activity}\left(\frac{d x(t)}{d t}\right)}{\operatorname{Activity}(x(t))}} \\
\operatorname{Complexity}(x(t))=\frac{\operatorname{Mobility}\left(\frac{d x(t)}{d t}\right)}{\operatorname{Mobility}(x(t))}
\end{gathered}
$$

$$
\text { HjorthIndex }=2 * \text { Complexity }+\frac{100}{2 * \text { Mobility }}
$$

where both mobility and complexity are averaged over all channels. 


\section{Data}

We have used the data set recorded at Derriford hospital (Plymouth, Devon, U.K.) used as evaluation data set in other recent studies [18][11][13]. It consists of $17 \mathrm{AD}$ cases $(9$ men and 8 women, age mean $=77.6$ years, std $=10)$ and 24 age-matched normal old (NOLD) cases (10 men and 14 women, age mean $=69.4$ years, std $=11.5)$. EEG were recorded from 21 scalp loci of the international 10-20 system with electrodes Fp1, Fp2, F7, F3, Fz, F4, F8, A1, T3, C3, Cz, C4, T4, A2, T5, P3, Pz, P4, T6, O1, O2 (See Figure 1). Each EEG is 4 minutes long (See Figure 2) and was left as raw as possible which would be the case for portable EEG machine at a GP surgery, with only bandpass filtering (0.5-50Hz IIR Chebyshev Type II filter) to remove DC and high frequency noise such as main sector.

\section{Results}

Results presented in this section are for each individual method and combined one using a fusion approach.

\subsection{Evaluation}

We use each method to calculate biomarkers per individual channel, then average them over all channels to obtain one single biomarker. With the results of all EEGs, we change the decision threshold and count True Positive (TP), True Negative (TN), False Positive (FP) and False Negative (FN) cases. We then calculate the sensitivity, calculated as the ratio of TP over $\mathrm{TP}+\mathrm{FN}$, the specificity, as the ratio of $\mathrm{TN}$ over $\mathrm{TN}+\mathrm{FP}$, and the accuracy (ratio of TP+TN over $\mathrm{TP}+\mathrm{TN}+\mathrm{FP}+\mathrm{FN}$ ). We also plot ROC curve for each method and calculate the Area Under the Curve (AUC) using the trapezoid rule and Standard Error (SE) [16]. Performance results are shown in Table 1 . We can clearly see that some methods are providing very high accuracy (such as FD, ZCI and $P w r_{\alpha, \theta}$ ) with both sensitivity and specificity better than $90 \%$ while others are not as robust.

As the size of our data set is relatively small $(\mathrm{N}=41)$, we prefer to plot the ROC curves showing the method's true performance with $95 \%$ confidence contours as shown in Figure 3(a) for FD, Figure 3(b) for ZCI and Figure 3(c) for $P w r_{\alpha, \theta}$.

\subsection{Fusion approach}

We investigated a fusion approach in which the outcome of each method are combined into one biomarker using a logistic regression model [21] of the form:

$$
y(X)=\frac{1}{1+e^{-\left(\beta_{0}+\sum_{i=1}^{N} \beta_{i} x_{i}\right)}}
$$

where $\beta_{0}$ is the bias, $\beta_{i}$ are the regression coefficients, and $x_{i}$ the values of each method. The overall performance, using the fusion approach, is improved to a $100.0 \%$ accuracy (sensitivity of $100 \%$ and specificity of $100 \%$ ). This shows the potential of combining multiple methods to obtain a more accurate early detection of AD rather than using individual method. The logistic regression model gives also indication on each method's discriminative power from a statistical point of view. Notice that this improvement is relative to the small number of cases $(\mathrm{N}=41)$ and such performance could decrease for larger data sets. Using the biomarker value of each method however, we can compare them on a case-to-case basis and help to assess which case was missed (i.e. False Negative) and thus further investigate.

\section{Conclusion}

In this paper we presented an evaluation study of promising methods for extracting biomarkers used for early detection of Alzheimer Disease (AD). The evaluation was carried out on data set with $17 \mathrm{AD}$ and 24 NOLD. We presented the performance results with each method's sensitivity, specificity, accuracy, AUC and SE. Due to the small sample size, ROC curves are plotted with the $95 \%$ confidence intervals are shown as contours.

To improve the diagnosis, we investigated a fusion approach, using each method biomarker as one feature of a logistic regression model. The final outcome was shown to improve the overall diagnosis accuracy (with the fusion outcome becoming perfect, i.e. AUC of 1.0). Moreover, such fusion approach provides insights on each method's discriminative power and allow to assess specific individual case missed by certain method. Ultimately, we can design an optimized classifier with only the best few methods. In this study they were found to be: FD, ZCI and $P w r_{\alpha, \theta}$.

The methods described in this paper have been developed in MATLAB. Work is in progress to port them in JAVA to further support online tools and services [15].

Future work will investigate the effects of artifacts removal and extend this evaluation to brain regions and frequency bands (delta, theta, alpha, beta, gamma, etc.), adding more methods like Lempel-Ziv complexity (LZ) [3], multiscale entropy (MSEnt) [1], and from information theory with LZW-based compression [33]. To further improve the clinical significance of our current evaluation, we will look at larger multi center data set [5] and possibly initiate EEG data collection from patients of different ethnic groups (e.g. from the Asia-Pacific region). 
Table 1. Performance results for all methods

\begin{tabular}{lccccc}
\hline \hline & Sen. & Spec. & Acc. & AUC & SE \\
\hline$P w r_{\alpha, \theta}$ & $94.12 \%$ & $91.67 \%$ & $92.68 \%$ & 0.975 & 0.027 \\
ZCI & $94.12 \%$ & $100.00 \%$ & $97.56 \%$ & 0.980 & 0.024 \\
FD & $94.12 \%$ & $100.00 \%$ & $97.56 \%$ & 0.989 & 0.018 \\
CTM & $47.06 \%$ & $79.17 \%$ & $65.85 \%$ & 0.605 & 0.091 \\
Hjorth & $64.71 \%$ & $66.67 \%$ & $65.85 \%$ & 0.620 & 0.091 \\
SamEnt & $29.42 \%$ & $79.17 \%$ & $63.41 \%$ & 0.561 & 0.092 \\
KolEnt & $82.35 \%$ & $50.00 \%$ & $63.41 \%$ & 0.577 & 0.092 \\
TsaEnt & $41.17 \%$ & $79.17 \%$ & $63.41 \%$ & 0.604 & 0.091 \\
\hline Fusion & $100.0 \%$ & $100.0 \%$ & $100.0 \%$ & 1.000 & \\
\hline \hline
\end{tabular}

\section{Acknowledgments}

This work was supported in part by BIOPATTERN project, EU Network of Excellence (FP6-2002-IST-1 No 508803).

\section{References}

[1] D. Abásolo, R. Hornero, J. Escudero, C. Gómez, M. García, and M. López. Approximate Entropy and Mutual Information Analysis of the Electroencephalogram in Alzheimers Disease Patients. MEDSIP, 27, 2006.

[2] D. Abásolo, R. Hornero, P. Espino, D. lvarez, and J. Poza. Entropy analysis of the EEG background activity in Alzheimer's disease patients. Physiological Measurement, 27(3):241-253, March 2006.

[3] D. Abásolo, R. Hornero, C. Gómez, M. García, and M. López. Analysis of EEG background activity in Alzheimer's disease patients with LempelZiv complexity and central tendency measure. Medical Engineering and Physics, 28(4):315-322, May 2006.

[4] K. Alloul, L. Sauriol, W. Kennedy, C. Laurier, G. Tessier, S. Novosel, and A. Contandriopoulos. Alzheimers disease: a review of the disease, its epidemiology and economic impact. Archives of Gerontology and Geriatrics, 27(3):189221, November 1998.

[5] C. Babiloni, G. Binetti, E. Cassetta, G. Dal Forno, C. Del Percio, F. Ferreri, R. Ferri, G. Frisoni, K. Hirata, B. Lanuzza, C. Miniussi, D. V. Moretti, F. Nobili, G. Rodriguez, G. L. Romani, S. Salinari, and P. M. Rossini. Sources of cortical rhythms change as a function of cognitive impairment in pathological aging: a multicenter study. Clinical Neurophysiology, 117(2):252-268, February 2006.

[6] C. Babiloni, E. Cassetta, G. Dal Forno, C. Del Percio, F. Ferreri, R. Ferri, B. Lanuzza, C. Miniussi, D. V. Moretti, F. Nobili, R. D. Pascual-Marqui, G. Rodriguez, G. L. Romani, S. Salinari, O. Zanetti, and P. M. Rossini. Donepezil effects on sources of cortical rhythms in mild Alzheimer's disease: Responders vs. Non-Responders. NeuroImage, 4(15):1650 1665, July 2006.

[7] B. S. Bloom, N. de Pouvourville, and W. L. Straus. Cost of illness of Alzheimer's disease: how useful are current estimates? Gerontologist, 43(2):158-164, April 2003.

[8] L. Bracco, R. Gallato, F. Grigoletto, A. Lippi, V. Lepore, G. Bino, M. P. Lazzaro, F. Carella, T. Piccolo, and C. Pozzilli. Factors affecting course and survival in Alzheimer's disease. A 9-year longitudinal study. Archives of Neurology, 51(12):1213-1219, 1994.

[9] E. Dishman and M. C. Carrillo. Perspective on everyday technologies for Alzheimers care: Research findings, directions, and challenges. Alzheimer's \& Dementia, 3(3):227234, July 2007.

[10] H. Forstl, H. Sattel, C. Besthorn, S. Daniel, C. GeigerKabisch, F. Hentschel, M. Sarochan, and R. Zerfass. Longitudinal cognitive, electroencephalographic and morphological brain changes in ageing and Alzheimer's disease. The British Journal of Psychiatry, 168:280-286, 1996.

[11] C. Goh, T. Cassar, C.-F. V. Latchoumane, C. Bigan, E. Ifeachor, G. Henderson, K. Camilleri, S. Fabri, J. Jeong, N. Hudson, P. Capotosto, S. Wimalaratna, and M. Besleaga. Comparison of Methods for Early Detection of Alzheimers Disease using Markers Derived from the EEG. . Submitted to Clinical Neurophysiology.

[12] C. Goh, E. Ifeachor, G. Henderson, C. V. Latchoumane, J. Jeong, C. Bigan, N. Hudson, M. Besleaga, P. Capotosto, and S. Wimalaratna. P04.3 Characterisation of EEG at different stages of Alzheimers disease (AD). Clinical Neurophysiology, 117(Supplement 1):138-139, September 2006. (Abstracts of the 28th International Congress of Clinical Neurophysiology).

[13] C. Goh, E. C. Ifeachor, T. Cassar, C.-F. Latchoumane, C. Bigan, G. Henderson, K. Camilleri, S. Fabri, J. Jeong, N. Hudson, P. Capotosto, S. Wimalaratna, and M. Besleaga. Comparison of Methods for Early detection of Alzheimer's disease. Proceedings of the 3rd International Conference on Computational Intelligence in Medicine and Healthcare (CIMED2007), Plymouth, U.K., July 25-27, 2007.

[14] J. H. Growdon. Biomarkers of Alzheimer Disease. Archives of Neurology, 56(3):281-283, March 1999.

[15] B. Hamadicharef, C. Goh, E. C. Ifeachor, C. Bigan, and N. Hudson. Online tools and services for early diagnosis and care for Alzheimers disease. Proceedings of the 3nd International Conference on Computational Intelligence in Medicine and Healthcare (CIMED2007), Plymouth, U.K., July 25-27, 2007.

[16] J. A. Hanley and B. J. McNeil. The Meaning and Use of the Area under a Receiver Operating Characteristic (ROC) Curve. Radiology, 143(1):29-36, 1982.

[17] L. E. Hebert, P. A. Scherr, J. L. Bienias, D. A. Bennett, and D. A. Evans. Alzheimer Disease in the US Population: Prevalence Estimates Using the 2000 Census. Archives of Neurology, 60(8):1119-1122, August 2003.

[18] G. Henderson, E. C. Ifeachor, N. Hudson, C. Goh, N. Outram, S. Wimalaratna, C. Del Percio, and F. Vecchio. Development and Assessment of Methods for Detecting Dementia using the Human Electroencephalogram. IEEE Transac- 


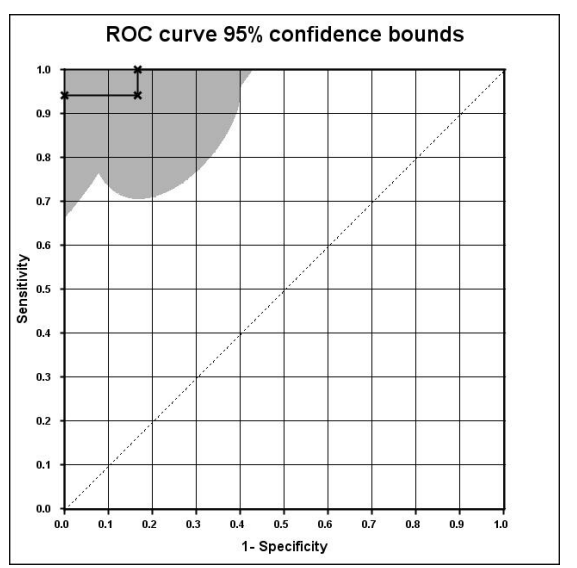

(a)

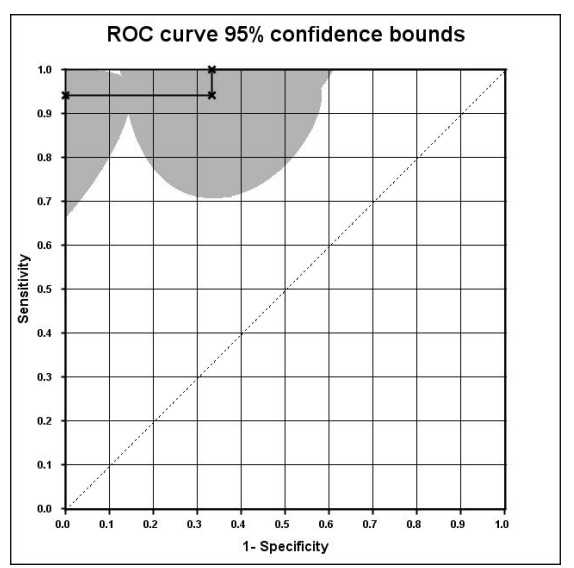

(b)

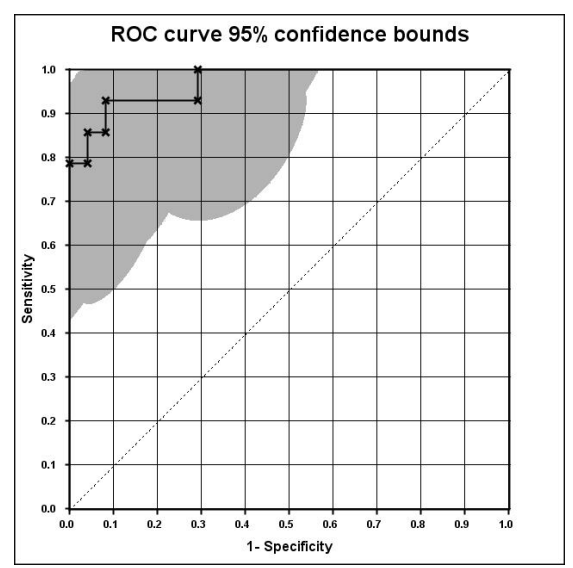

(c)

Figure 3. ROC curve for (a) FD, (b) ZCI and (c) $P w r_{\alpha, \theta}$ method

tions on Biomedical Engineering, 53(8):1557-1568, August 2006.

[19] G. Henderson, E. C. Ifeachor, S. Wimalartna, E. Allen, and N. Hudson. Prospects for routine detection of dementia using the fractal dimension of the human electroencephalogram. Proceedings of the 1st International Conference on Advances in Medical Signal and Information Processing (MEDSIP2000), Bristol, U.K., September 4-6, 2000, pages 284-289.

[20] B. Hjorth. EEG analysis based on time domain properties. Electroencephalography and Clinical Neurophysiology, 29(3):306-310, September 1970.

[21] D. W. Hosmer and S. Lemeshow. Applied Logistic Regression: Wiley Series in Probability and Statistics. New York, NY, John Wiley and Sons, September 2000. IBSN 0471356328.

[22] E. C. Ifeachor, N. J. Outram, G. T. Henderson, H. Wimalaratna, N. Hudson, R. Sneyd, C. Dong, and C. Bigan. Non-linear methods for biopatern analysis: role and challenges. Proceedings of the 26th Annual International Conference of the IEEE Engineering in Medicine and Biology Society (EMBS'04), San Francisco, USA, September 1-5, 2004.

[23] J. Jeong. EEG dynamics in patients with Alzheimer's disease. Clinical Neurophysiology, 115(7):1490-1505, July 2004.

[24] F. Kaspar and H. G. Schuster. Easily Calculable Measure for the Complexity of Spatiotemporal Patterns. Physical Review A, 36(2):842-848, July 1987.

[25] B. Leifer. Early Diagnosis of Alzheimer's Disease: Clinical and Economic Benefits. Journal of the American Geriatrics Society, 51(5):281-288, May 2003.

[26] P. McNamee, J. Bond, and D. Buck. Costs of Dementia in England and Wales in the 21st century. The British Journal of Psychiatry, 179:261-266, 2001.

[27] S. G. Post. Future Scenarios for the Prevention and Delay of Alzheimer Disease Onset in High-Risk Groups: An Ethical Perspective. American Journal of Preventive Medicine, 16(2):105-110, February 1999.
[28] U. Schreiter-Gasser, T. Gasser, and P. Ziegler. Quantitative EEG analysisin early onset Alzheimer's disease: a controlled study. Electroencephalography and Clinical Neurophysiology, 86(1):15-22, January 1993.

[29] H. Soininen, J. Partanen, V. Laulumaa, E.-L. Helkala, M. Laakso, and P. J. Riekkinen. Longitudinal EEG spectral analysis in early stage of Alzheimers disease. Electroencephalography and Clinical Neurophysiology, 72:290-297, 1989.

[30] H. Soininen, J. Partanen, V. Laulumaa, A. Paakkonen, E. L. Helkala, and P. J. Riekkinen. Serial EEG in Alzheimer's disease: 3 year follow-up and clinical outcome. Electroencephalography and Clinical Neurophysiology, 79:342-348, 1991.

[31] E. Souetre, R. M. Thwaites, and H. L. Yeardley. Economic impact of Alzheimer's disease in the United Kingdom. Cost of care and disease severity for non-institutionalised patients with Alzheimer's disease. The British Journal of Psychiatry, 174:51-55, 1999.

[32] J. Wancata, R. Musalek, Alexandrowicz, and M. Krautgartner. Number of dementia sufferers in Europe between the years 2000 and 2050. European Psychiatry, 18(6):306-313, October 2003.

[33] P. Zhao and E. C. Ifeachor. EEG assessment of Alzheimers diseases using universal compression algorithm. Proceedings of the 3rd International Conference on Computational Intelligence in Medicine and Healthcare (CIMED2007), Plymouth, U.K., July 25-27, 2007.

[34] P. Zhao, P. Van Eetvelt, C. Goh, N. Hudon, S. Wimalaratna, and E. C. Ifeachor. EEG markers of Azheimer's disease using Tsallis entropy. Proceedings of the 3rd International Conference on Computational Intelligence in Medicine and Healthcare (CIMED2007), Plymouth, U.K., July 25-27, 2007. 